\title{
MECHANICAL LOSS SPECTRUM OF THE Fe-20\%Ni-C ALLOYS AT NEGATIVE TEMPERATURES
}

\author{
Stoyan Parshorov*, and IVAn Parshorov \\ Institute of Metal Science Equipment and Technologies \\ with Hydro and Aerodynamics Centre "Acad. Angel Balevski", \\ Bulgarian Academy of Sciences \\ 67, Shipchenski Prohod Blvd, 1574, Sofia, Bulgaria, \\ e-mails: st_parshorov@ims.bas.bg; parsh@ims.bas.bg
}

\begin{abstract}
The paper considers investigation of the mechanical loss spectrum of $\mathrm{Fe}-20 \%$ (mass) $\mathrm{Ni}-\mathrm{C}$ in the temperature range 100-300 K by means of the X-ray and internal friction method. At temperatures of the order of $250 \mathrm{~K}$ relaxation maximum is observed with relaxation parameters - activation energy $H=10000$ $[\mathrm{cal} / \mathrm{mol}]$ and relaxation constant $\tau_{0}^{250} 6.15 \times 10^{-11}[\mathrm{~s}]$. The maximum has behaviour similar to that of the Bordoni maximum in alloys with face centered cubic lattice related to the fundamental properties of the dislocations. The relationship established between the values of the microstructural parameters of martensite and the hardening temperatures showed that there is a certain critical size of the dislocation segments determined by the carbon concentration, below which the relaxation mechanisms defined by Seeger and Pare are not realized.

Keywords: X-ray, internal friction, mechanical loss spectrum, relaxation maximum, relaxation parameters, dislocations.
\end{abstract}

\section{INTRODUCTION}

According to the state diagram for the alloy Fe-20\% (mass) Ni-C it is characteristic that:

- At room temperature it is martensitic, i.e. there is a start martensite point above room temperature [1];

- When cooling, the austenite-martensitic phase trasformation proceeds in a non-diffusion path at a low rate [2-4], in contrast to non-alloy or low-alloy alloys, in which this trasformation proceeds at a rate close to that of light;

\footnotetext{
${ }^{*}$ Corresponding author.

DOI: 10.7546/EngSci.LVIII.21.01.04
} 
- Unlike high-alloy manganese alloys with very low energy of stacking defects and martensitic reaction of type $\gamma \Rightarrow \varepsilon$, nickel alloys have relatively higher energy of stacking defects and are characterized by the conversion of type $\gamma \Rightarrow \alpha[5]$

- The basis of the mechanism of $\gamma \Rightarrow \alpha$ martensitic phase transformation lies in the dislocation microstructure and the presence of point defects in the solid solution and the formed atomic configuration architecture of the iron atoms of the solvent and those of nickel and carbon [5].

The aim of the present article is to shed light on the behaviour of the highly stressed and rich in structural defects matrix through the integrated methods of research - internal friction and X-ray diffraction analysis.

\section{MATERIAL AND METHODOLOGY}

The alloy iron $-20 \%$ nickel, is made in a vacuum furnace from materials of high purity. The casting is first forged and then rolled into tiles with a thickness of $0.8 \mathrm{~mm}$ and $3 \mathrm{~mm}$. The samples for examination of internal friction, with dimensions $1.5 \times 0.8 \times 55 \mathrm{~mm}$, and those for X-ray diffraction analysis, with dimensions $15 \times 15 \times 3 \mathrm{~mm}$, are obtained by electrospark cutting. They are subjected to purification in a hydrogen atmosphere, after which some of them are subjected to carbon in a medium of methane and hydrogen, ensuring the presence of $135 \mathrm{ppm}$ of carbon.

The internal friction test is performed with highly sensitive and fully automated equipment at operating frequencies in the range of 1-3 Hz. The relative working deformation is in the range $10^{-5}-10^{-6}$, guaranteeing the operation of the pendulum in the amplitude-independent region. The tests are performed under cooling and subsequent heating at a rate of $2{ }^{\circ} \mathrm{C} / \mathrm{min}$ in the temperature range $100-700 \mathrm{~K}$. In this range, when heating to $300 \mathrm{~K}$, the $\gamma \Rightarrow \alpha$ phase trasformation is excluded. Thus, the dynamic relaxation spectrum includes only effects caused by the structural states of the solid solution.

The kinetic parameters of relaxation are determined at three operating frequencies. The known dependence of the Arenius type is used, in which $\tau$ relaxation time $[\mathrm{s}], T$-absolute temperature $[\mathrm{K}], H$-activation energy $[\mathrm{cal} / \mathrm{mol}]$, $\tau_{0}$-relaxation constant [s] and $R$-fundamental gas constants [cal $\left./ \mathrm{mol} . \mathrm{K}\right]$ are related to the dependence [5]:

$$
\tau=\tau_{0} \cdot \exp (H / R T) .
$$

According to the methodology described in detail in [5], the analysis of their relaxation maxima is performed. It is based on the principle that if 
the width of the relaxation maximum is greater than that corresponding to a relaxation time of the process, then there is a distribution of relaxation times with a distribution parameter $\beta$. X-ray diffraction analysis involves imaging the diffraction lines $(111)_{\gamma}$ of austenite and $(110)_{\alpha}$ and $(200)_{\alpha}$ of martensite under $F e_{K a}$ radiation. According to the standard method, based on the comparison of the intensity of the diffraction lines of austenite and martensite $(111)_{\gamma}$ and $(110)_{\alpha}$, respectively, [6], it was found that the amount of rest austenite after quenching in all test specimens is within $0-8 \%$.

An X-ray diffraction analysis is performed based on the width of two Xray diffraction lines [7]. The total integral width of each line includes the instrumental width of the line and the expansion caused by microstructural inhomogeneities of martensite, such as: size of the crystal domains $D$ and the absolute value of the microdeformation of the lattice $<\varepsilon^{2}>^{1 / 2}$. Each microstructural parameter gives its share in the expansion of each of the studied diffraction lines to a degree depending on its size and the crystallographic direction of the respective reflection line.

The samples for both types of tests are hardened at temperatures of 680 , 740,800 and $900{ }^{\circ} \mathrm{C}$ from the austenitic region in quartz ampoules in argon atmosfere, in water.

\section{EXPERIMENTAL RESULTS}

Figure 1 shows the experimental results obtained for the investigated alloy after quenching from different temperatures.

In the temperature range $200-300 \mathrm{~K}$, a relaxation maximum is observed at a temperature of the order of $250 \mathrm{~K}$ depending on the operating frequency. It was found that:

- The relaxation maximum is reversible on heating and cooling, which shows that it is not associated with irreversible processes, such as phase transformations.

- The height of the relaxation maximum depends on the hardening temperature, Fig. 1.

- The relaxation maximum is also observed after quenching in non-carbon samples, as it is greater and wider than that of carbon samples quenched by the same temperature, Fig. 2. This shows that carbon does not cause the relaxation process, but only quantitatively affects it through some microstructural parameter.

The determined maximum kinetic parameters of relaxation are $H=10000$ $[\mathrm{cal} / \mathrm{mol}]$ and $\tau_{0}^{250}=6.15 \times 10^{-11}$ [s], Fig. 2 . 


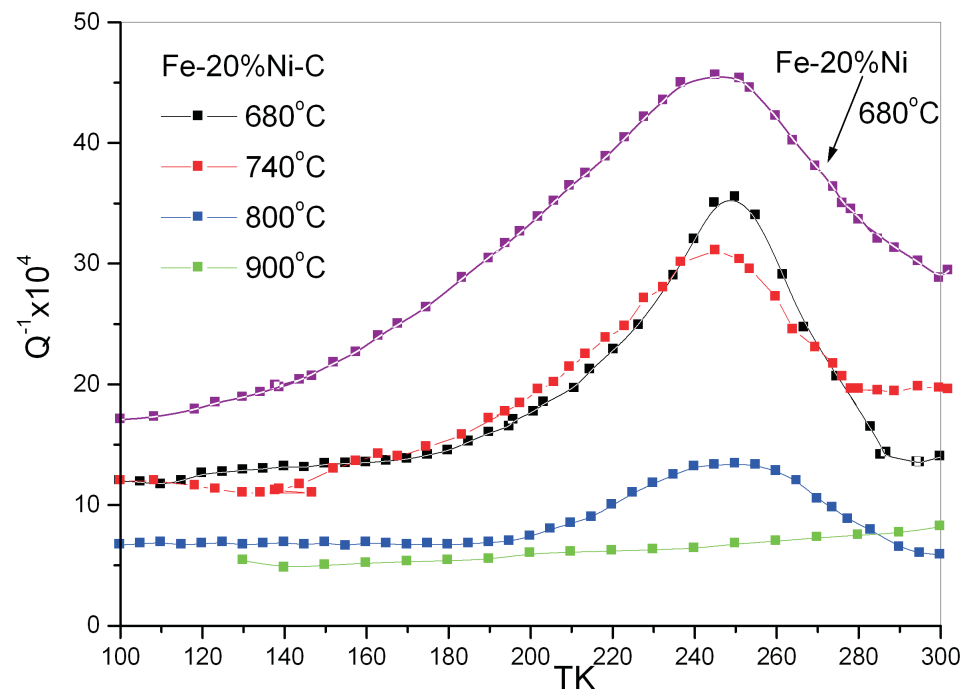

Fig. 1. Loss spectrum (internal friction) at different hardening temperatures (test temperature range 100-300 K)

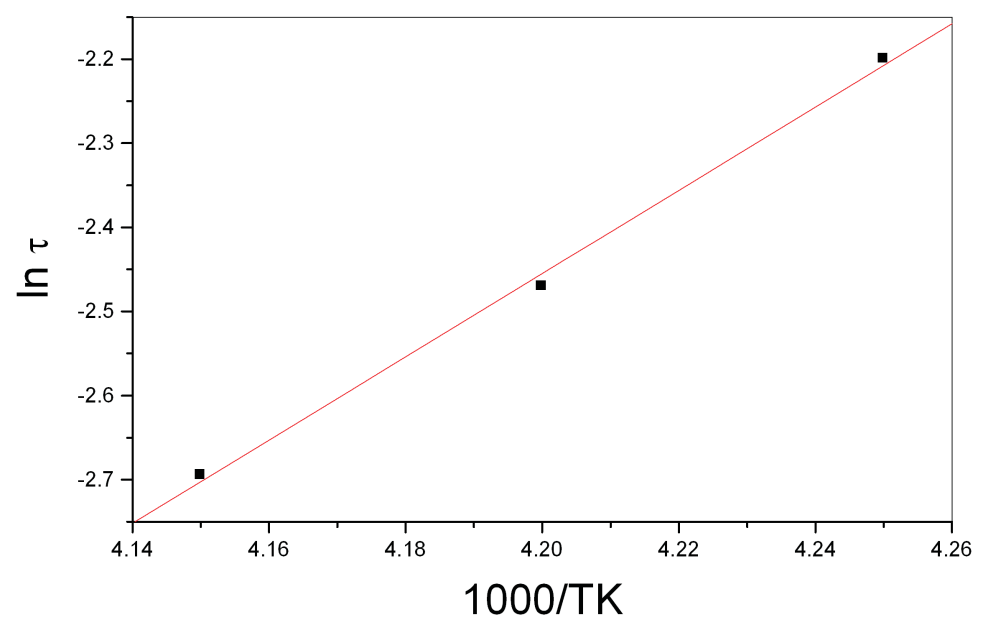

Fig. 2. Determination of the kinetic parameters of the relaxation maximum

The calculated values of the distribution parameter of the relaxation time are shown in Fig. 3 and given in Table 1. It was found that the presence of carbon and the lower hardening temperature lead to a decrease in distribution parameter $\beta$. 


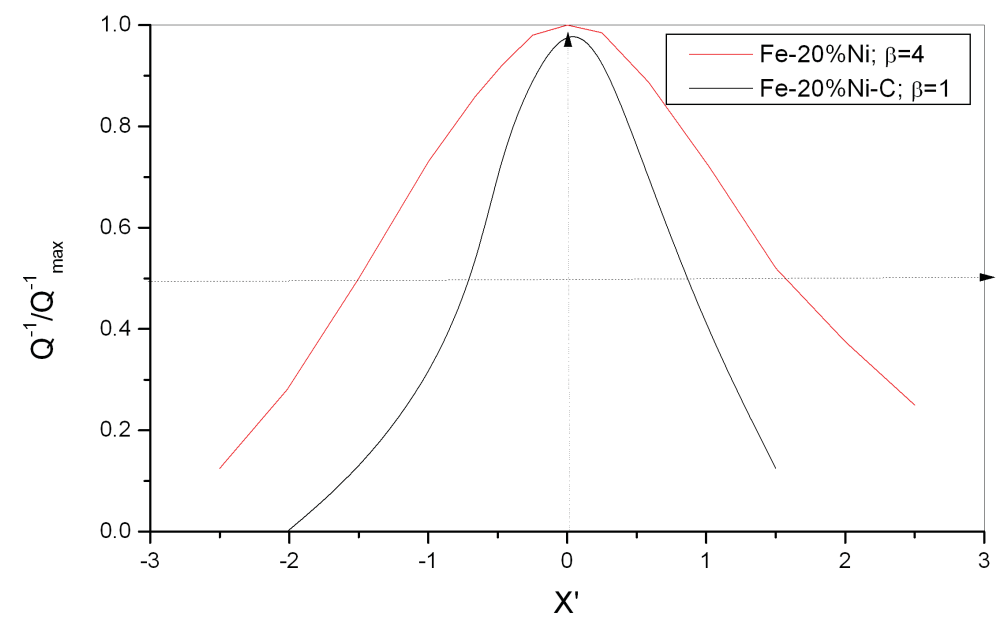

Fig. 3. Determination of the distribution parameter for Fe-Ni and Fe-Ni-C alloys hardened by $680^{\circ} \mathrm{C}$

Table 1. Values of the distribution parameter of relaxation times $\beta$ at different hardening temperatures

\begin{tabular}{|c|c|c|c|c|c|}
\hline Alloy & Fe- $20 \% \mathrm{Ni}$ & \multicolumn{4}{|c|}{$\mathrm{Fe}-20 \% \mathrm{Ni}-\mathrm{C}$} \\
\hline Hardening temperature & $680{ }^{\circ} \mathrm{C}$ & $680{ }^{\circ} \mathrm{C}$ & $740{ }^{\circ} \mathrm{C}$ & $800{ }^{\circ} \mathrm{C}$ & $900{ }^{\circ} \mathrm{C}$ \\
\hline$\beta$ & 4.0 & 1.0 & 1.275 & 1.5 & 1.75 \\
\hline
\end{tabular}

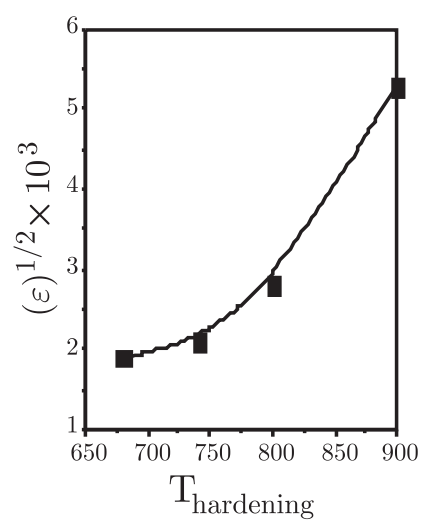

(a)

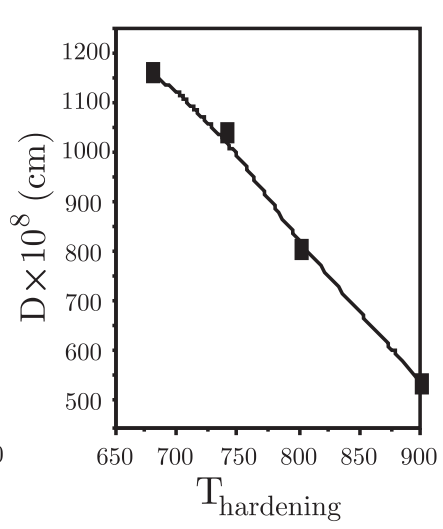

(b)

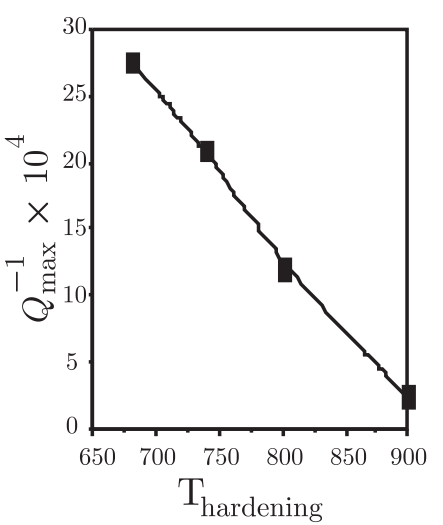

(c)

Fig. 4. (a) Dependence of the microdeformation $<\varepsilon^{2}>^{1 / 2}$;

(b) the size of the crystal domains $D$; (c) the heights of the relaxation maxima $Q_{\max }^{-1}$ on the hardening temperature 
Studies show that the values of microstresses $<\varepsilon^{2}>^{1 / 2}$, the size of the crystal domains $D$ and the height of the relaxation maximum $Q^{-1}$ max depend on the hardening temperature, Fig. $4(\mathrm{a}-\mathrm{c})$. As the hardening temperature increases, the microdeformation of the crystal lattice increases, and the size of the domains and the heights of the relaxation maxima decrease. The quenching of the samples in air and water does not lead to any noticeable change in the microstructural parameters of the martensite, which shows that they are mainly influenced by the temperature and not by the rate of quenching.

\section{DISCUSSION OF THE EXPERIMENTAL RESULTS}

The first studies of the internal friction in iron-nickel alloys (Ni 17\%) with $0.02 \%$ carbon $[8,9]$ revealed the presence of $\alpha$-relaxation maximum of martensite after hardening. The authors arrive at a conclusion that this maximum is caused by the presence of internal stresses. Similarly to our research, the reversibility of the relaxation process has been established.

For metals with Body Centered Cubic (BCC) lattice at low temperatures, so-colled $\alpha$ - and $\beta$-maxima are known. The kinetic parameters of relaxation published in the literature for them can be summarized as follows: activation energy for $\alpha$-maximum $H=0.18-0.25 \mathrm{eV}$ (approximately $4.1-5.6 \mathrm{kcal} / \mathrm{mol}$ ), and for $\beta$-maximum $K=0.4-0.47 \mathrm{eV}$ (approximately $9.0-10.7 \mathrm{kcal} / \mathrm{mol}$ ), at time constant for both maxima $\tau_{0}=10^{-11}-10^{-12}[\mathrm{~s}][5]$.

It is argued [10-15] that the $\alpha$-maximum is analogous to the Bordoni maximum and $\beta$ to the Hasiguti maximum in Face Centered Cubic (FCC) metals, respectively.

It is necessary to know that despite everything, there is no exact interpretation of the dislocation maxima in BCC metals for now.

All the above characteristics of the low-temperature maximum indicate that it is most likely caused by relaxation associated with the fundamental properties of dislocations. This is guaranteed by the relatively low energy activation and the relatively large value of the relaxation constant and the strong influence of the height of the maximum of the internal stresses and the presence of atoms of incorporation in the martensite.

Our experimental data show that this is a relaxation similar to or a variant of Bordoni, which is confirmed by the following facts:

1. The two relaxation maxima are not established in heated samples. Our research has shown that heating to temperatures in the range of 400-500

$\mathrm{K}$ leads to the complete disappearance of the maximum.

2. Retention at room temperature, at a temperature of minus $20^{\circ} \mathrm{C}$, and at

Engineering Sciences, LVIII, 2021, No. 1 
the temperature of the liquefied nitrogen does not lead to change or disappearance of the relaxation maximum.

3. Both relaxations are not conditioned by embedding atoms in the crystal lattice.

4. The presence of impurity atoms reduces the height of the relaxation maximum.

5. The values obtained by us for the kinetic parameters of relaxation of the maximum Fig. 2 and those of the Bordoni maximum in BCC metals are close. These are processes with relatively low activation energy and low flow rate, which is typical for dislocation relaxation.

6 . The values of the time constant $\tau_{0}$ show that it is not a question of relaxation of point defects, which are characterized by significantly higher speeds of the processes.

7. Analogously to our research, the reversibility of the relaxation process has been established. From a physical point of view, the Bordoni maximum is best explained by the model of A. Seeger [16-18]. The Seeger model involves the appearance on the dislocation line of a pair of steps with an inverse sign with a critical distance between them, which move in opposite directions under the action of the applied voltage. The shortcomings in Seeger's theory are related to the fact that only dislocation lines parallel to the Peierls-Nabarro valleys take part in the relaxation process [19-22]. The probability of forming a pair of steps of critical size and the probability that these steps will move away rather than to be annihilated remain unexplained.

V. Pare [23] solves the problems related to Seeger's theory, taking into account the influence of internal stresses and their orientation towards the dislocation line in the energetic relief of the formation of pairs of steps. It is assumed that the internal stresses are favorably oriented towards the dislocation segments relative to the Peierls-Nabarro valleys. This quantifies the degree of relaxation obtained. The length of the dislocation segment is also included in Pare's theory as a decisive factor for the realization of the relaxation process.

Our research shows that as the hardening temperature increases, the internal stresses increase. This leads to a reduction of the allowable length of the dislocation segments, which can take part in the relaxation process, hence the parameter of the distribution of relaxation times increases. In an alloy without carbon, the greater width of the relaxation maximum, as well as its greater height, can be explained by the greater length of the dislocation segments than those of carbon martensite. Since there is a correlation between 
the length of the dislocation segments and the size of the domains, it can be concluded that as the hardening temperature increases, the dislocation density and internal stresses increase, and the lengths of the dislocation segments decrease the relaxation maximum, as after hardening of $900{ }^{\circ} \mathrm{C}$ it leads to the disappearance of the relaxation maximum. Therefore, below a certain critical size of the dislocation segment, the relaxation mechanism associated with the Seeger and Pare models is not realized.

\section{CONCLUSION}

The studies of the temperature dependence of internal friction in the temperature range $100-300 \mathrm{~K}$ of $\mathrm{Fe}-20 \% \mathrm{Ni}$ alloy can be summarized as follows:

- At temperatures of the order of $250 \mathrm{~K}$ of the temperature dependence of internal friction on hardened samples of $\mathrm{Fe}-20 \% \mathrm{Ni}$ alloy, the presence of a relaxation maximum was established with relaxation parameters - activation energy $H=10000[\mathrm{cal} / \mathrm{mol}]$ and relaxation constant $\tau_{0}^{250}=6.15 \times 10^{-11}[\mathrm{~s}]$.

- The maximum has behaviour similar to that of the Bordoni maximum in alloys with FCC lattice, related to the fundamental properties of the dislocations.

- The relationship established between the values of the microstructural parameters of martensite and the hardening temperatures shows that there is a certain critical size of the dislocation segments determined by the carbon concentration, below which the relaxation mechanisms defined by Seeger and Pare are not realized.

\section{ACKNOWLEDGEMENTS}

The authors are grateful to the financial support of Bulgarian National Science Fund at the Ministry of Education and Science, Contract No. DN17/17 /12.12.2017/.

All equipment and experimental units used in this work was funded by the European Regional Development Fund within the OP "Science and Education for Smart Growth 2014-2020", project CoE "National center of mechatronics and clean technologies", No. BG05M2OP001-1.001-0008-C08. 


\section{REFERENCES}

[1] M. Hansen, Constitution of Binary alloys, $2^{\text {nd }}$ Edition, McGraw-Hill Companies (1958).

[2] Y. Petrov, Defects and non-diffusion transformation into steel, Kiev, Naukovaya Dumka (1978).

[3] J. Guimarães And P. Riosa, Acta Materialia (2015) $\mathbf{8 4} 436$, https://doi.org/10.1016/j.actamat.2014.10.04.

[4] J. Guimarâes And P. Riosa, Journal of Materials Research and Technology (2018) 7 (4) 499, https://doi.org/10.1016/j.jmrt.2018.04.007.

[5] A. Nowick And B. Berry, Anelastic Relaxation in Crystalline Solids, Academic Press, New York and London (1972).

[6] S. Gorelik, Y. Skakov, And L. Rastorguev, X-ray and electron-optical analysis, MISIS (2002).

[7] G. Faninger and F. Sturm, Materialprufung (1969) 11 (1) 381.

[8] A. Menschikov, Physics of Metals and Metals Science (1975) 40 (4) 853.

[9] H. Chang and S. Sastri, Pergamon press (1979) 131047.

[10] W. Benoit, Mechanical Spectroscopy $Q^{-1}$ with Applications to Materials Science, Ch. 3.1 Dislocation - Description and Dynamics, Trans Tech Publications LTD (2001) 141.

[11] W. Benoit, Mechanical Spectroscopy $Q^{-1}$ with Applications to Materials Science, Ch. 3.2 Dislocation - Lettice Interactions, Trans Tech Publications LTD (2001) 158 .

[12] Mikhail S. Blanter, Igor S. Golovin, Hartmut Neuhäuser, and HansRainer Sinning, Internal Friction in Metallic Materials, Handbook, SpringerVerlag, Berlin-Heidelberg, Springer Series in Materials Science, eBook (2007) 542, ISBN 978-3-540-68758-0, DOI: 10.1007/978-3-540-68758-0.

[13] P. Pal-Val, L. Pal-Val, A. Rybalko, and E. Vatazhuk, Change of Parameters of the Koiwa-Hasiguti Dynamic Dislocation Relaxation in Nanostructured and Polycrystalline Zirconium after Severe Plastic Deformation and Annealing, Advances in Materials Science and Engineering (2018) 1, DOI: $10.1155 / 2018 / 4170187$.

[14] W. Wasserbäch, S. Sahling, R. Pohl, and E. Thompson, LowTemperature Internal Friction and Thermal Conductivity of Plastically Deformed, High-Purity Monocrystalline Niobium, Journal of Low Temperature Physics (2002) 127 (3) 121-151, DOI: 10.1023/A: 1014848312572.

[15] V. NAtsik and Yu. Semerenko, Dislocation mechanism of low-temperature internal friction in nanostructured materials, Low Temperature Physics (2016) 42 (2) 138, DOI: 10.1063/1.4942907.

[16] A. SeEger, Philosophical Magazine (1956) 1651.

[17] A. SeEger, Journal de Physique (1971) 32 C2-193. 
[18] A. Seeger, Journal de Physique (1981) 42 C5-201.

[19] G. Costantini and F. Marchesoni, Internal Friction Peaks at Low Temperatures: a Kink Model Analysis, Journal de Physique IV (Proceedings) (1996) 6 (8) C8-187, DOI: 10.1051/jp4:1996838.

[20] M. Bujard, G. Gremaud, J. Baur, and W. Benoit, Kink pair formation mechanism (KPF) studied in aluminium by cycle bias stress experiments, Journal de Physique (1985) C10 (46) 325, DOI:10.1051/jphyscol:19851072.

[21] R. Peierls, Physical Society (1940) 5234.

[22] R. Nabarro, Advances in Physics (1952) 1269.

[23] V. PARE, Journal of Applied Physics (1961) 32332.

Received February 25, 2021

Engineering Sciences, LVIII, 2021, No. 1 